INPLASY

PROTOCOL

To cite: Zhou et al. Efficacy and safety of Chinese herbal medicine Xiao Yao San in menstruation disturbances: a systematic review and metaanalysis. Inplasy protocol

202140053. doi:

10.37766/inplasy2021.4.0053

Received: 09 April 2021

Published: 09 April 2021

Corresponding author:

Yaping Wang

ypwang30@163.com

Author Affiliation:

Formula-Pattern Research Center, School of Traditional Chinese Medicine, Jinan

University

Support: ZH2020KF01; 2020B1111100001.

Review Stage at time of this submission: Piloting of the study selection process.

\section{Efficacy and safety of Chinese herbal medicine Xiao Yao San in menstruation disturbances: a systematic review and meta-analysis}

Zhou, X1; Ma, Q2; Yang, N3; Lin, S4; Wang, Y5; Chen, J6; Wang, Y7.

Review question / Objective: 1. Are the Chinese herbal medicine, Xiao Yao San, effective in treating menstruation disturbances? 2. Is it safe to use Xiao Yao San for the treatment of menstruation disturbances?

Condition being studied: Menstrual disorders are a disruptive physical and/or emotional symptoms just before and during menstruation, including heavy bleeding, missed periods and unmanageable mood swings.

Information sources: Comprehensive search will be carried out in seven electronic databases, including MEDLINE, Embase, the Cochrane Library, Chinese Biomedical Database (CBM), China National Knowledge Infrastructure (CNKI), Wanfang Database, China Science and Technology Journal Database (VIP). No publication date or language restriction will be imposed.

INPLASY registration number: This protocol was registered with the International Platform of Registered Systematic Review and Meta-Analysis Protocols (INPLASY) on 09 April 2021 and was last updated on 09 April 2021 (registration number INPLASY202140053).

Conflicts of interest:

None declared.

\section{INTRODUCTION}

Review question / Objective: 1. Are the Chinese herbal medicine, Xiao Yao San, effective in treating menstruation disturbances? 2. Is it safe to use Xiao Yao
San for the treatment of menstruation disturbances?

Condition being studied: Menstrual disorders are a disruptive physical and/or emotional symptoms just before and during 
menstruation, including heavy bleeding, missed periods and unmanageable mood swings.

\section{METHODS}

Participant or population: Women patients with menstrual disorder symptoms who attain Xiao Yao San or modified Xiao Yao san alone or with western medicines, will be included. No restriction on age, gender, or nationality. Women patients who had surgery for gynecological neoplasms, pelvic inflammatory disease, or using an intra-uterine contraceptive device will be excluded.

Intervention: Trials that compared Xiao Yao San or modified Xiao Yao San as the active intervention in the treatment group versus placebo or conventional medicines will be included. Trials will be excluded if any other medications, including qigong, Tai Chi, acupuncture, cupping, moxibustion, and massage, were used as co-interventions. The treatment duration should be at least 2 weeks.

Comparator: Placebo or conventional medicines for menstruation disturbances.

Study designs to be included: Randomized controlled trials.

Eligibility criteria: Inclusion criteria: studies will be eligible for inclusion if they meet all of the following five criteria: (1) patients were diagnosed with menstruation disturbances either by a clinician or according to specific diagnostic criteria; (2) studies were conducted as RCTs; (3) effects of Xiao Yao San (XYS) or modified Xiao Yao San (MXYS) in treating FD were assessed; (4) the possible comparisons were as follows: XYS or MXYS vs. placebo, XYS or MXYS plus conventional medicines vs. conventional medicines, XYS or MXYS plus conventional medicines vs. placebo; (5) efficacy evaluation criteria were sufficiently described; and (6) treatment lasted for at least 4 weeks. Exclusion criteria: studies will be excluded if they meet any of the following criteria: (1) patients who had surgery for gynecological neoplasms, pelvic inflammatory disease, or using an intra-uterine contraceptive device; (2) patients could not localize their discomfort; (3) pregnant or lactating women were involved; and (4) XYS or MXYS was combined with other traditional therapies such as qigong, Tai Chi, acupuncture, cupping, moxibustion, and massage.

Information sources: Comprehensive search will be carried out in seven electronic databases, including MEDLINE, Embase, the Cochrane Library, Chinese Biomedical Database (CBM), China National Knowledge Infrastructure (CNKI), Wanfang Database, China Science and Technology Journal Database (VIP). No publication date or language restriction will be imposed.

\section{Main outcome(s): Response rate.}

Additional outcome(s): Quality of life, sleep disorders, estrogenic hormone level, anxiety, depression, adverse events.

Data management: Selection of studies: Two reviewers will independently screen the titles, abstracts and manuscripts retrieved from different databases engines and identify studies that potentially meet the inclusion criteria outlined above. Study selection and exclusion process will be documented and a list of potentially eligible studies in the analysis will be created. The full text of these potentially eligible studies will be downloaded and further assessed for eligibility by the two reviewers. Any disagreements will be resolved by a third reviewer as necessary. The reasons for the exclusion of any studies will be recorded on a PRISMA flow chart. Data extraction: Data extraction will be performed by two independently reviewers using a predesigned collection form. If any discrepancies arise, an additional reviewer will be consulted. The following information will be extracted for each study: (1) Publication characteristics; (2) Participants characteristics; (3) Interventions: intervention, comparison, concomitant medications, duration of treatment and 
follow-up time. (4) Outcomes: primary and secondary outcomes and adverse events.

Quality assessment / Risk of bias analysis: The methodological quality of each study was assessed by Dr. Xuan Zhou and Dr. Qingyu Ma according to criteria from the Cochrane risk of bias, including random sequence generation (selection bias), allocation concealment (selection bias), blinding of participants and personnel (performance bias), blinding of outcome assessment (detection bias), incomplete outcome data (attrition bias), selective reporting (reporting bias) and other bias. Any discrepancies will be resolved by Dr. Wang.

Strategy of data synthesis: Statistical analyses will be performed using the Cochrane RevMan software (version 5.3.0, Copenhagen, the Nordic Cochrane Center). Dichotomous data will be presented as relative risk (RR) or odds ratio (OR) with 95\% confidence intervals (Cls). The $X^{2}$ test will be used to evaluate the heterogeneity and $I^{2}$ will be used to assess the inconsistency across studies. Values of $\mathrm{I}^{2}$ ranged from 0 to $100 \%\left(I^{2}<40 \%\right.$, might not be important; $30 \%<\mathrm{I}^{2}<60 \%$, moderate heterogeneity; $50 \%<\mathrm{I}^{2}<90 \%$, substantial heterogeneity; $75 \%<I^{2}<100 \%$, considerable heterogeneity). Fixed-effect model will be used to pool estimates. Potential sources of heterogeneity will be identified by subgroup analysis and sensitivity analysis. Potential publication bias will be assessed graphically with funnel plot.

Subgroup analysis: Subgroup analyses will be conducted according to interventions in trial group and in control group.

Sensitivity analysis: Sensitivity analysis will be undertaken to examine if the effects are modified by, or robust to, the type of measurement method or tool.

Language: No language limits will be imposed.

Country(ies) involved: China.
Other relevant information: None.

Keywords: Menstruation disturbances, Xiao Yao San, meta analysis.

Contributions of each author:

Author 1 - Xuan ZHOU.

Author 2 - Qingyu MA.

Author 3 - Nating YANG.

Author 4 - Shaoqin LIN.

Author 5 - Yanan WANG.

Author 6 - Jiaxu CHEN.

Author 7 - Yaping WANG. 